\title{
Tracking Underwater Noise Sources with the Use of a Passive Method
}

\author{
I. GlozA*, K. Buszman And R. JózWiak \\ Polish Naval Academy, ul. Śmidowicza 69, 81-103 Gdynia, Poland
}

\begin{abstract}
It is possible to track a source of noise by determining the intensity of sound that informs about the sound energy flow direction. One of the methods of determining the sound intensity of acoustic signals is the so-called gradient method in which the pressure is measured with the use of two hydrophones placed close to each other. Tetrahedral antenna consists of four hydrophones spaced at regular tetrahedron vertices so that distances between hydrophones are the same. The tetrahedral antenna determines six virtual points in the middle of each edge and allows using sound intensity calculation algorithm to determine the noise source bearing. In our experiment, the antenna was located 23 meters below the sea surface and a source of noise was moving over it. The object that was moving along a straight line with a specific course was equipped with a GPS receiver. This allowed comparison of the obtained targeting results with the real track of moving object. The paper presents the object tracking algorithm which was implemented in the MATLAB environment as well as some of the results of research carried out on passive source noise tracking.
\end{abstract}

DOI: 10.12693/APhysPolA.123.1090

PACS: $43.30 . \mathrm{Nb}$

\section{Introduction}

It is an important issue to track objects that are the source of noise and move on the water surface using passive systems. Due to the nature of the application, solutions to this problem remain unpublished in general. Passive measurement system consumes significantly less power than similar active systems. Due to batterypowered measurement system, it can carry out measurements without having the battery recharged for longer time. In addition, such a system is noiseless during the measurement, so the object being tracked cannot detect its presence.

Passive positioning system that is designed by the Marine Technology Centre of Polish Naval Academy in Gdynia is a complementary measurement module. The system has been developed by authors of this publication. There are four hydrophones placed on the frame creating a regular tetrahedron - tetrahedral antenna [1]. The lengths of all edges of the tetrahedron are the same, and the distances between the acoustic centers of hydrophones are set up to $250 \mathrm{~mm}$.

In this arrangement of hydrophones one can set six virtual hydrophones that are placed in the middle of segments connecting the two hydrophones (edges of the tetrahedron). The pair of virtual hydrophones corresponding to the $V 2-V 4$ is $0 X$ axis, $V 5-V 6$ is $0 Y$ axis, and $V 1-V 3$ is $0 Z$ axis in Cartesian coordinates system. Origin of the Cartesian coordinate system is determined by the point of intersection of lines connecting each pair of virtual hydrophones $V 1-V 3, V 2-V 4, V 5-V 6$, as shown in Fig. 1.

\footnotetext{
*corresponding author; e-mail: i.gloza@amw.gdynia.pl
}

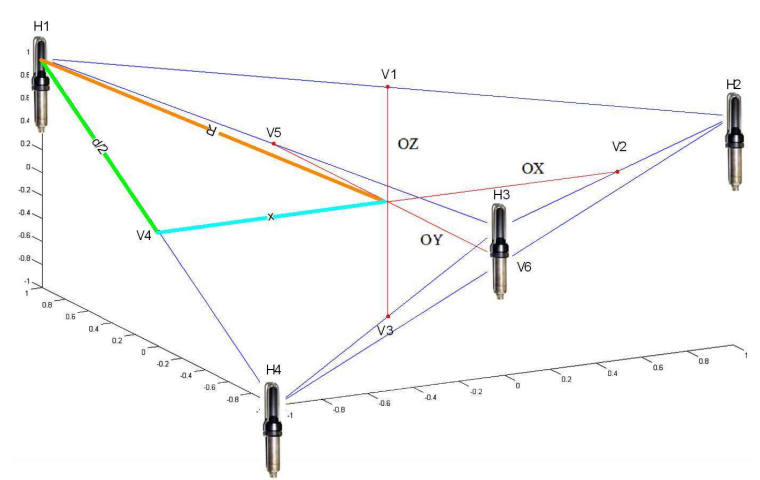

Fig. 1. Arrangement of hydrophones and virtual points with triangle in a tetrahedron.

Distances between virtual hydrophones can be determined with respect to the radius of the sphere described on the tetrahedron as shown in Fig. 1.

The radius $R$ of the sphere described on the regular tetrahedron is:

$$
R=\frac{\sqrt{6}}{4} d,
$$

where $d$ is the distance between hydrophones, $x$ is a half of the distance between virtual hydrophones, and $R$ is a radius of the sphere described on the regular tetrahedron.

Distance $x$ between virtual hydrophones depending on the sides length of a triangle can be determined from equation:

$$
2 x=\frac{d}{\sqrt{2}} .
$$

The distance between virtual hydrophones is $250 \mathrm{~mm} \times$ $2^{1 / 2}=176.8 \mathrm{~mm}$. 


\section{The research method}

If the distance between virtual hydrophones is known, the virtual signals corresponding to virtual hydrophones can be calculated.

Phase shifts and amplitudes of signals received by hydrophones depend on the location of the noise source. Figure 2 shows the signals received by the four hydrophones for one run of noise source over the measuring module. Signals were filtered by the narrowband filter with a center frequency $f_{0}=631 \mathrm{~Hz}$ and bandwidth $B=100 \mathrm{~Hz}$.

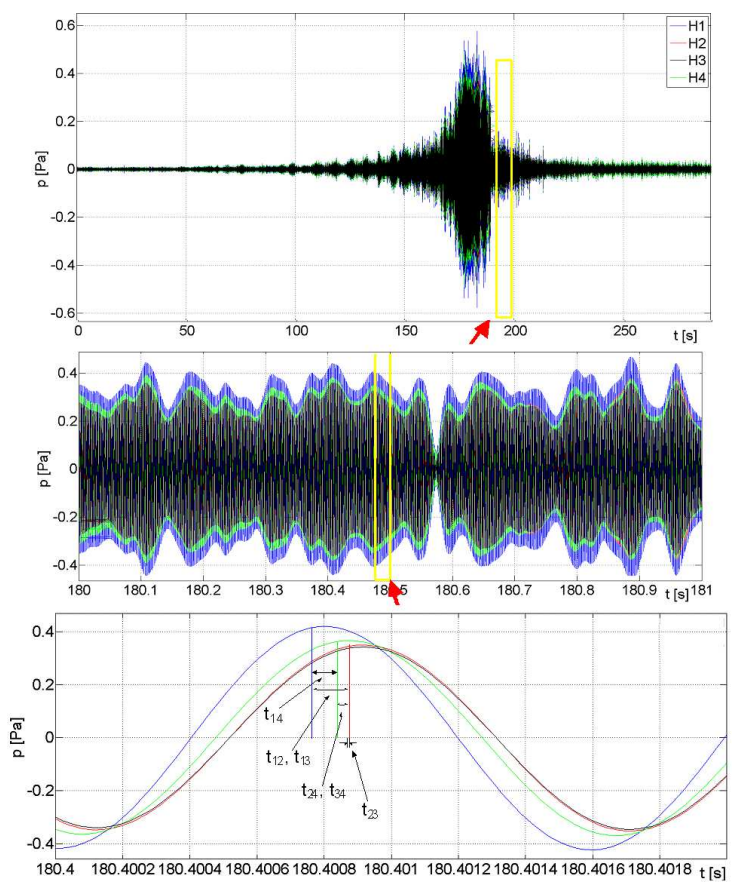

Fig. 2. Acoustic pressure change in time, $t_{n m}$ is the time shift between signals $H_{n}$ and $H_{m}$.

In order to determine the signal for one of virtual hydrophones, the time shift between selected pair of hydrophones that is assigned to a virtual signal has to be specified. The next step is to move the selected signal by a delay set so that both signals were consistent in phase. Further, the resultant signal shifted by a half length of the calculated delay is to be obtained.

The best example illustrating calculation of a virtual signal is the signal $V_{1}$ designation located between hydrophones $\mathrm{H} 1$ and $\mathrm{H} 2$. Determination of the time delay between $H_{1}$ and $H_{2}$ begins with the autocorrelation function calculation of the hydrophone signal $H_{1}$ :

$$
R_{H_{1}}(\tau)=\int_{-\infty}^{\infty} H_{1}(t) H_{1}^{*}(t-\tau) \mathrm{d} t .
$$

The cross-correlation function of signals $H_{1}$ and $H_{2}$ is shown in Fig. 3:

$$
R_{H_{1} H_{2}}(\tau)=\int_{-\infty}^{\infty} H_{1}(t) H_{2}^{*}(t-\tau) \mathrm{d} t .
$$

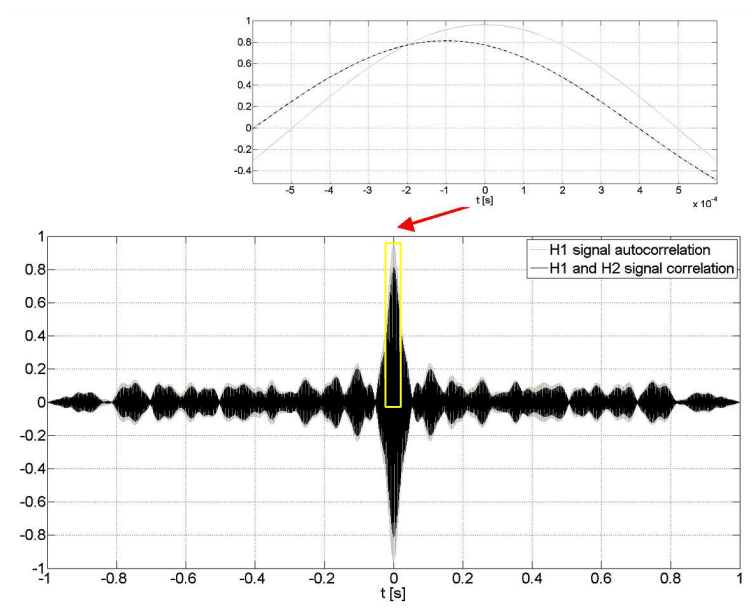

Fig. 3. Correlation of signals $H_{1}$ and $H_{2}$.

At first maxima of the autocorrelation and cross correlation functions were obtained. In the next step, locations of the maxima were specified that allows the time shift $\tau$ between signals from the hydrophones $\mathrm{H} 1$ and $\mathrm{H} 2$ to be specified. The signal from the hydrophone $\mathrm{H} 2$ is shifted by the determined delay in the previous step so that the signals of $H_{2}$ and $H_{1}$ are in coinciding phases:

$$
H_{2}^{\prime}(t)=H_{2}(t \pm \tau) \text {. }
$$

Then the resultant signal $V_{1}^{\prime}$ is determined as

$$
V_{1}^{\prime}(t)=\frac{\left.H_{1} t\right)+H_{2}^{\prime}(t)}{2} .
$$

Finally, the virtual signal $V_{1}$ is determined by the signal $V_{1}^{\prime}$ delayed by $\tau / 2$ :

$$
V_{1}(t)=V_{1}^{\prime}\left(t \mp \frac{\tau}{2}\right) \text {. }
$$

The determined signal $V_{1}^{\prime}$ shifted by $\tau / 2$, since the virtual hydrophone is located in the middle of section between hydrophones $\mathrm{H} 1$ and $\mathrm{H} 2$.

After determining all six virtual signals, three components of sound intensity $I_{x}, I_{y}$, and $I_{z}$ must be determined. For this purpose the cross spectrum function was used as follows $[2,3]$.

$$
\begin{gathered}
I_{x}=-\frac{\operatorname{Im}\left\{G_{V_{4} V_{2}}\right\}}{2 \rho \omega x}, \\
I_{y}=-\frac{\operatorname{Im}\left\{G_{V_{5} V_{6}}\right\}}{2 \rho \omega x}, \\
I_{z}=-\frac{\operatorname{Im}\left\{G_{V_{1} V_{3}}\right\}}{2 \rho \omega x},
\end{gathered}
$$

where $\rho$ is water density, $\omega=2 \pi f$ is the analyzed signal pulsation ( $f$ being the signal frequency), $2 x=d / 2^{1 / 2}$ is the distance between virtual points ( $d$ being the distance between hydrophones), and $\operatorname{Im} G_{V_{A} V_{B}}$ is the imaginary part of the cross spectrum. 


\section{Measurement results}

The experiment was carried out in the Baltic Sea [4]. The measuring module (Fig. 4) was located at a depth of 23 meters with integrated tetrahedral antenna and a digital compass allowing subsequent correction of the position of the antenna relative to the north. Object representing a source of noise was moving over the antenna with a specified course and a speed of 6 knots. The object track was recorded by the GPS receiver so that it was possible to compare the calculated route of the tracking object with the real track.

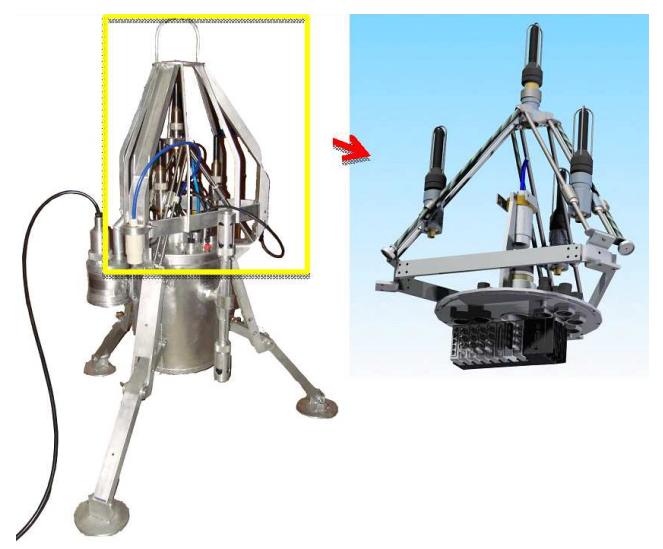

Fig. 4. The measuring module.

The algorithm allows specifying a single bearing of the object, so it is necessary to divide the signal recorded into the short time periods available for analysis. For determination of a temporary bearing, 1-second periods were chosen because for these time intervals most accurate results were obtained.

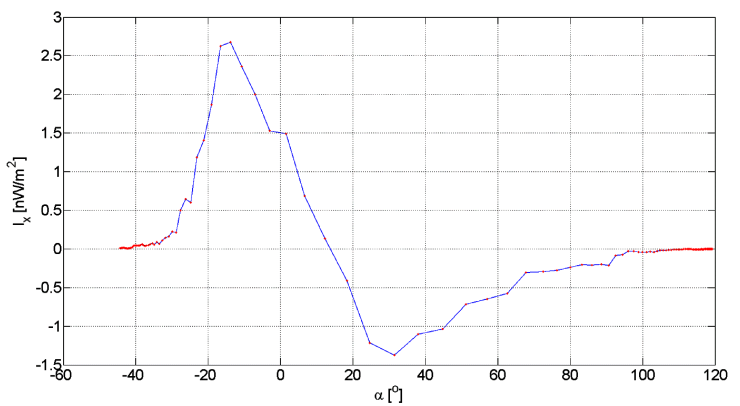

Fig. 5. $I_{x}$ component of the sound intensity as a function of bearing angle $\alpha$.

If the intensity components $I_{x}, I_{y}$, and $I_{z}$ (Figs. 5-7) are known, it is possible to determine the source of the noise bearing angle in three dimensions according to the formula

$$
\varphi=\arctan \left(\frac{I_{y}}{I_{x}}\right) .
$$

The determined bearing angle $\varphi$ can be seen in Fig. 8 .

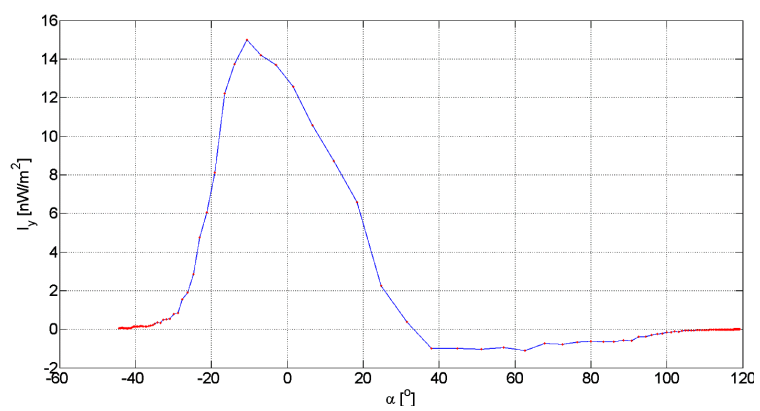

Fig. 6. $I_{y}$ component of the sound intensity as a function of bearing angle $\alpha$.

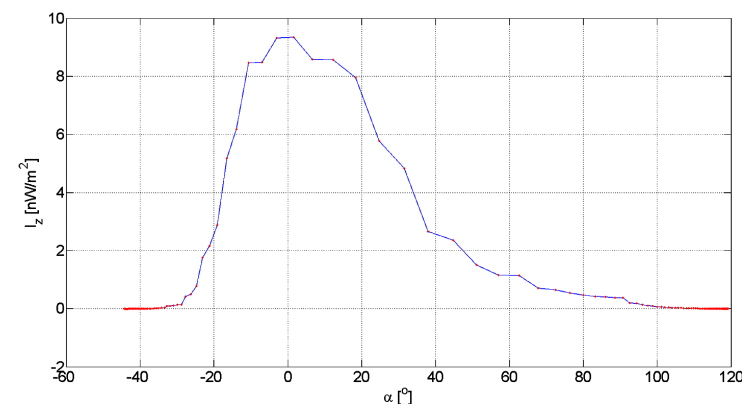

Fig. 7. $I_{z}$ component of the sound intensity as a function of bearing angle $\alpha$.

It is possible to change coordinates to rectangular, assuming a known distance from the object $r$ and $\theta=90^{\circ}$ :

$$
\begin{aligned}
& x=r \sin \theta \cos \varphi, \\
& y=r \sin \theta \sin \varphi, \\
& z=r \cos \theta .
\end{aligned}
$$

The first targeting result was obtained directly from components of the determined intensity. The following quantities were taken into account: the depth of the mounted unit, moment of time when the position of the source was over the measurement module, and the determined speed of the object. The $x$-axis was directed on a course $310^{\circ}$. The value was read from a digital compass. It was

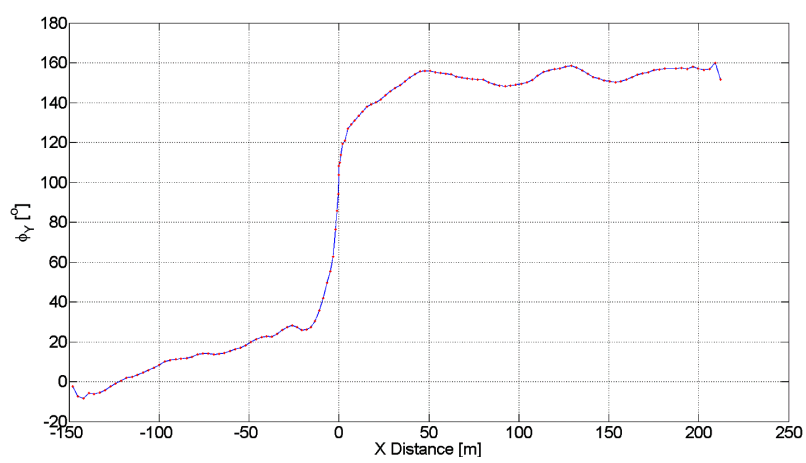

Fig. 8. Angle $\varphi$ as a function of distance. 
necessary to do this in order to rotate the result by $50^{\circ}$ as can be seen in Fig. 9 .

$$
\left[\begin{array}{lll}
X^{\prime} & Y^{\prime} & Z^{\prime}
\end{array}\right]=\left[\begin{array}{lll}
X & Y & Z
\end{array}\right]\left[\begin{array}{ccc}
\cos (\alpha) & \sin (\alpha) & 0 \\
-\sin (\alpha) & \cos (\alpha) & 0 \\
0 & 0 & 1
\end{array}\right]
$$

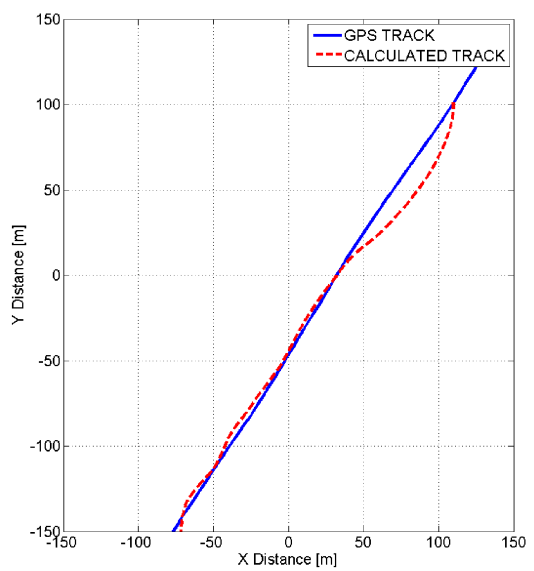

Fig. 9. The calculated rotated track.

\section{Conclusions}

The algorithm presented in this paper allows for the passive tracking of a moving noise source on the water surface [5-7]. The sound intensity method of bearing calculation of moving target provides sufficient accuracy for small distances from the target in the shallow water. Bearing was verified with the actual position obtained from the GPS receiver. The advantage of using algorithm is the possibility to track a source in real time. It is expected to carry out tests using noise sources under the surface. To do this, the module will be equipped with underwater navigation system which will allow the comparison of the tetrahedral antenna system bearing with underwater navigation.

\section{Acknowledgments}

The investigation was supported by the Ministry of Science and Higher Education (Grant No OR00 0028 12).

\section{References}

[1] M. Kastek, R. Dulski, M. Życzkowski, M. Szustakowski, P. Trzaskawka, W. Ciurapinski, G. Grelowska, I. Gloza, S. Milewski, K. Listewnik, Proc. SPIE 8388, 83880M-1 (2012).

[2] F.J. Fahy, Sound intensity, 2nd ed., E\&FN spon, London 1995.

[3] I. Gloza, Acta Acustica \&S Acustica 88, 670 (2002).

[4] G. Grelowska, Archiv. Acoust. 25, 359 (2000).

[5] D. Ross, Trends in Merchant Shipping (1969 - 1980), Tetra Tech. Inc. Report, TT-SD 449-75-1 (April 1975).

[6] P.T. Arveson, D.T. Vendittis, J. Acoust. Soc. Am. 107, 118 (2000).

[7] E. Kozaczka, J. Domagalski, G. Grelowska, I. Gloza, Polish Maritime Research 14, 40 (2007). 